\title{
Correction to: Relaxation Dynamics of Ethanol and N-Butanol in Diesel Fuel Blends from Terahertz Spectroscopy
}

Rayda Patiño-Camino ${ }^{1}$ - Alexis Cova-Bonillo ${ }^{1}$ José Rodríguez-Fernández ${ }^{1}$. Teresa P. Iglesias ${ }^{2} \cdot$ Magín Lapuerta $^{1}$

Published online: 14 October 2021

(c) Springer Science+Business Media, LLC, part of Springer Nature 2021

Correction to: Journal of Infrared, Millimeter and Terahertz Waves. (2021) 42:772-792 https://doi.org/10.1007/s10762-021-00807-5

Table 1 should read as follows:

Table 2 should read as follows:

The original article can be found online at https://doi.org/10.1007/s10762-021-00807-5.

Magín Lapuerta

Magin.Lapuerta@uclm.es

1 Escuela Técnica Superior de Ingeniería Industrial, Edificio Politécnico Universidad de CastillaLa Mancha, Avda. Camilo José Cela S-N, 13071 Ciudad Real, Spain

2 Departamento de Física Aplicada, Facultad de Ciencias Universidad de Vigo, 36200 Vigo, Spain 
Table.1 Most common modifications from Debye model

\begin{tabular}{ll}
\hline Model & Equation \\
\hline Debye [19] & $\varepsilon(\omega)=\varepsilon_{\infty}+\frac{\varepsilon_{s}-\varepsilon_{\infty}}{1+i \omega \tau}$ \\
Cole-Cole [23] & $\varepsilon(\omega)=\varepsilon_{\infty}+\frac{\varepsilon_{s}-\varepsilon_{\infty}}{1+(i \omega \tau)^{1-\alpha}}$ \\
Davidson-Cole [24] & $\varepsilon(\omega)=\varepsilon_{\infty}+\frac{\varepsilon_{s}-\varepsilon_{\infty}}{(1+i \omega \tau)^{\beta}}$ \\
Havriliak-Negami [25] & $\varepsilon(\omega)=\varepsilon_{\infty}+\frac{\varepsilon_{s}-\varepsilon_{\infty}}{\left(1+(i \omega \tau)^{1-\alpha}\right)^{\beta}}$ \\
\hline
\end{tabular}

$\alpha, \beta$ are in the range $[0,1]$

Table.2 Expansion of the real and imaginary parts of the Debye dielectric relaxation model for diesel, biodiesel, ethanol, and n-butanol

\begin{tabular}{|c|c|}
\hline Diesel & $\begin{array}{l}\varepsilon_{\text {realD }}(\omega)=\varepsilon_{\infty D}+\frac{\varepsilon_{1 D}-\varepsilon_{\infty D}}{1+\left(\omega \cdot \tau_{1 D}\right)^{2}} \\
\varepsilon_{\text {imgD }}(\omega)=\frac{\left(\varepsilon_{1 D}-\varepsilon_{\infty D}\right) \cdot \omega \cdot \tau_{1 D}}{1+\left(\omega \cdot \tau_{1 D}\right)^{2}}\end{array}$ \\
\hline Biodiesel & $\begin{array}{l}\varepsilon_{\text {realB }}(\omega)=\varepsilon_{\infty B}+\frac{\varepsilon_{1 B}-\varepsilon_{2 B}}{1+\left(\omega \cdot \tau_{1 B}\right)^{2}}+\frac{\varepsilon_{2 B}-\varepsilon_{\infty B}}{1+\left(\omega \cdot \tau_{2 B}\right)^{2}} \\
\varepsilon_{\text {imgB }}(\omega)=\frac{\left(\varepsilon_{1 B}-\varepsilon_{2 B}\right) \cdot \omega \cdot \varepsilon_{1 B}}{1+\left(\omega \cdot \tau_{1 B}\right)^{2}}+\frac{\left(\varepsilon_{2 B}-\varepsilon_{\infty B}\right) \cdot \omega \cdot \varepsilon_{2 B}}{1+\left(\omega \cdot \tau_{2 B}\right)^{2}}\end{array}$ \\
\hline Ethanol & $\begin{array}{l}\varepsilon_{\text {realEt }}(\omega)=\varepsilon_{\infty E t}+\frac{\varepsilon_{1 E t}-\varepsilon_{2 E t}}{1+\left(\omega \cdot \tau_{1 E t}\right)^{2}}+\frac{\varepsilon_{2 E t}-\varepsilon_{3 E t}}{1+\left(\omega \cdot \tau_{2 E t}\right)^{2}}+\frac{\varepsilon_{3 E t}-\varepsilon_{\infty E t}}{1+\left(\omega \cdot \tau_{3 E t}\right)^{2}} \\
\varepsilon_{\text {imgEt }}(\omega)=\frac{\left(\varepsilon_{1 E t}-\varepsilon_{2 E t}\right) \cdot \omega \cdot \tau_{1 E t}}{1+\left(\omega \cdot \tau_{1 E t}\right)^{2}}+\frac{\left(\varepsilon_{2 E t}-\varepsilon_{3 E t}\right) \cdot \omega \cdot \tau_{2 E t}}{1+\left(\omega \cdot \tau_{2 E t}\right)^{2}}+\frac{\left(\varepsilon_{3 E t}-\varepsilon_{\infty E t}\right) \cdot \omega \cdot \tau_{3 E t}}{1+\left(\omega \cdot \tau_{3 E t}\right)^{2}}\end{array}$ \\
\hline n-Butanol & $\begin{array}{l}\varepsilon_{\text {realBu }}(\omega)=\varepsilon_{\infty B u}+\frac{\varepsilon_{1 B u}-\varepsilon_{2 B u}}{1+\left(\omega \cdot \tau_{1 B u}\right)^{2}}+\frac{\varepsilon_{2 B u}-\varepsilon_{3 B u}}{1+\left(\omega \cdot \tau_{2 B u}\right)^{2}}+\frac{\varepsilon_{3 B u}-\varepsilon_{\infty \circ B}}{1+\left(\omega \cdot \tau_{3 B u}\right)^{2}} \\
\varepsilon_{i m g B u}(\omega)=\frac{\left(\varepsilon_{1 B u}-\varepsilon_{2 B u}\right) \cdot \omega \cdot \tau_{1 B u}}{1+\left(\omega \cdot \tau_{1 B u}\right)^{2}}+\frac{\left(\varepsilon_{2 B u}-\varepsilon_{3 B u}\right) \cdot \omega \cdot \tau_{2 B u}}{1+\left(\omega \cdot \tau_{2 B u}\right)^{2}}+\frac{\left(\varepsilon_{3 B u}-\varepsilon_{\infty B u}\right) \cdot \omega \cdot \tau_{3 B u}}{1+\left(\omega \cdot \tau_{3 B u}\right)^{2}}\end{array}$ \\
\hline
\end{tabular}

Publisher's Note Springer Nature remains neutral with regard to jurisdictional claims in published maps and institutional affiliations. 